\title{
Three-Tesla Magnetic Resonance and Computed Tomography Imaging in Three-Dimensional Conformal Radiotherapy for Localized Prostate Cancer
}

\author{
Gianluca Ingrosso*, Alessandra Carosi, Elisabetta Ponti, Pierluigi Bove, Riccardo Santoni \\ Department of Diagnostic Imaging, Molecular Imaging, Interventional Radiology and Radiotherapy, \\ Tor Vergata University General Hospital, Italy \\ Email: gianluca.ingrosso@libero.it
}

Received August 19, 2011; revised September 18, 2011; accepted September 27, 2011

\begin{abstract}
Aims and background: we evaluate CT-3Tesla MRI fusion in conformal radiotherapy for localized prostate cancer.Methods: 18 consecutive patients underwent a 3T MRI scan under radiotherapy planning conditions, after the CT scan. Bowel and bladder preparation were prescribed. CT and MR images were automatically fused; prostate and seminal vesicles were contoured on CT and on MRI, organs at risk were defined on CT-MRI fusion. Late rectal and sexual toxicity, differences in target volume between MRI and CT and differences in rectal and penile bulb dose distribution based on CT only or on CT-MRI fusion were evaluated.Results: one patient experienced a late rectal toxicity; no patient had sexual toxicity. The difference between the mean MRI and CT target volumes was statistically significant ( $\mathrm{p}=0.0001$ paired Student's t-test). The dose-volume histogram (DVH) analysis shows a significant reduction of the dose received by the rectum and the penile bulb in MRI-plans compared to CT-plans.Conclusions: 3 Tesla MRI scan under radiotherapy planning conditions along with bowel preparation significantly improves the definition of the target volume sparing normal tissue irradiation.
\end{abstract}

Keywords: Prostate Cancer, 3 Tesla Magnetic Resonance, Computed Tomography, Conformal Radiotherapy

\section{Introduction}

Prostate cancer is the most common male malignancy in Western countries and the second leading cause of cancer death in men $[1,2]$. The improvement of the screening has lead to the identification of prostate cancer at an earlier and potentially treatable stage and three dimensional conformal radiation therapy (3DCRT), along with surgery and brachytherapy, are the standard therapies for localized prostate cancer [3-8]. In 3DCRT the accurate delineation of the clinical target volume (CTV) is crucial in particular with intensity-modulated radiation therapy (IMRT), where it is possible to increase the radiation dose to the target volume, sparing at the same time the surrounding normal tissues and minimizing the risk of acute and late complications [9-11]. Computed tomography $(\mathrm{CT})$ scanning is the most common approach to localize the prostate in radical conformal radiotherapy, but it has poorer soft-tissue contrast than magnetic resonance imaging (MRI), in particular when differentiating the prostate gland from the periprostatic soft tissues. Three-Tesla MRI allows excellent morphologic information of prostate, penile bulb anatomy, and of rectumprostate interface [12-13].

In this study we retrospectively evaluated 3DCRT based on CT-3Tesla MR image fusion, for 18 patients with localized prostate cancer. Differences in CTV (prostate + seminal vesicles) volume between MRI and CT and differences in terms of rectal and penile bulb dose distribution based on CT only or CT-MRI fusion were evaluated as well as late rectal and sexual toxicity.

\section{Materials and Methods}

Eighteen patients with localized prostate cancer underwent CT-planned radical 3D conformal radiotherapy at the Radiation Oncology Therapy Unit of the University of Rome Tor Vergata; after a 3T MRI scan, under radiotherapy planning conditions, to evaluate the feasibility of CT-3T MRI image registration in order to define 
prostate CTV. This study was approved by the hospital ethics committee, and all of the 18 patients were asked to sign a written informed consent agreement regarding the use of a 3T MRI.

Patient characteristics are summarized in Table 1. The median age at the time of treatment was 72 years (range 46-78 years); 13 patients were classified as T1c clinical stage, 4 were T2a and 1 patient T2c (TNM, American Joint Committee on Cancer 2002). The median PSA (prostate specific antigen; normal values range between 0 $\mathrm{ng} / \mathrm{ml}$ and $4 \mathrm{ng} / \mathrm{ml}$ ) value at diagnosis was $9.39 \mathrm{ng} / \mathrm{ml}$ (range 5.21 - $42 \mathrm{ng} / \mathrm{ml}$ ), and the Gleason score (a grading system that assigns a grade to each of the two largest areas of cancer in the tissue samples, grades range from 1 to 5) was $6(3+3)$ in 9 patients, $7(3+4)$ in 2 cases and $7(4+3)$ in 1 case, $8(4+4)$ in 5 patients and $9(4+5)$ in 1 patient; 10 / 18 cases were treated with 6 months hormone-releasing hormone agonist in association with radiotherapy. Nine patients had erectile dysfunction before the start of radiotherapy / hormone therapy, due to age, diabetes and cardio-vascular co-morbidities.

Bowel preparation was obtained suggesting a diet in combination with a daily mild laxative to reduce intestinal gas and obtain a reproducible bowel volume during
CT and MRI acquisition and treatment sessions. For bladder preparation patients were asked to empty their bladder for better daily prostate localization.

CT scanning was performed with a GE LightSpeed ${ }^{\circledR}$ Scanner (GE Healthcare Diagnostic Imaging, Slough, UK). The scan was to start at the level of the iliac crests and continue down through the perineum, with a $2.5 \mathrm{~mm}$ slice thickness. MRI study took place within 20 minutes after CT scanning and was performed with a 3.0 T Philips Achieva Intera (Philips Medical Systems, Reigate, UK); T2 TSE (turbo-spin eco) weighted images were acquired with the following scan parameters: repetition time $(\mathrm{TR})=1000 \mathrm{~ms}$, echotime $(\mathrm{TE})=90 \mathrm{~ms}$, TSE factor $=16, \mathrm{FOV}=400 \times 400 \mathrm{~mm}$, acquisition matrix $304 \mathrm{x}$ 240 reconstructed to $512 \times 512$, slice thickness $2.5 \mathrm{~mm}$, $50-60$ slices, number of signal averages $(\mathrm{NSA})=2$; total acquisition time: 8 - 10 minutes. Patients were scanned, both for CT and MRI, supine on a flat couch top with the arms on the chest; ankle stocks were used to prevent rotation of the hips, and localizing tattoos were used to maintain a stable position. For 3 T MRI a homemade wood couch insert was used to achieve a flat scan surface.

Table 1. Patients characteristics $(n=18)$.

\begin{tabular}{|c|c|c|c|c|}
\hline & median & range & & n. of patients \\
\hline Age & $72 \mathrm{y}$ & $46 y-78 y$ & & \\
\hline \multirow[t]{2}{*}{ PSA at diagnosis } & $9.39 \mathrm{ng} / \mathrm{ml}$ & $5.21 \mathrm{ng} / \mathrm{ml}-42 \mathrm{ng} / \mathrm{ml}$ & & \\
\hline & & & $\mathrm{T} 1 \mathrm{c}$ & 13 \\
\hline \multirow[t]{4}{*}{ T-stage } & & & $\mathrm{T} 2 \mathrm{a}$ & 4 \\
\hline & & & $\mathrm{T} 2 \mathrm{c}$ & 1 \\
\hline & & & $6(3+3)$ & 9 \\
\hline & & & $7(3+4)$ & 2 \\
\hline \multirow[t]{3}{*}{ Gleason score } & & & $7(4+3)$ & 1 \\
\hline & & & $8(4+4)$ & 5 \\
\hline & & & $9(4+5)$ & 1 \\
\hline \multirow{4}{*}{$\begin{array}{l}\text { Sexual dysfunction } \\
\text { before RT/hormone- }\end{array}$} & & & yes & 9 \\
\hline & & & & \\
\hline & & & no & 9 \\
\hline & & & yes & 10 \\
\hline \multicolumn{5}{|l|}{ Hormone-therapy } \\
\hline & & & no & 8 \\
\hline
\end{tabular}


CT and MRI images were exported on Syntegra soft ware (Pinnacle, Philips Medical System, Andover, MA) and the two data sets were automatically fused; Syntegra is a multi-modality image registration software that provides manual and point-based image registration, and three automated methods of gray value-based image registration: cross correlation, local correlation and mutual information. The result of each registration was always checked by a physician, inspecting visually the matching of bony structures and soft tissues.

For each patient the clinical target volume (CTV) and organs at risk (OARs) were outlined by the same radiation oncologist. The target volume irradiated to $66 \mathrm{~Gy}$ $\left(\mathrm{CTV}_{1}\right)$ consisted of prostate and seminal vesicles; the boost irradiated to $76 \mathrm{~Gy}\left(\mathrm{CTV}_{2}\right)$ was the prostate only.
Planning target volumes $\left(\mathrm{PTV}_{1}\right.$ and $\left.\mathrm{PTV}_{2}\right)$ were generated by an asymmetric expansion of CTVs $(6 \mathrm{~mm}$ in all directions except at the posterior margin, where a $4 \mathrm{~mm}$ expansion was used). The target volumes were delineated on CT images and on MRI images afterwards (Figure 1); the CTV contouring for each of the two set of images was performed blindly with respect to the other one.

The rectum was contoured on CT-MRI fusion as solid organ from the $8^{\text {th }}$ slice $(2 \mathrm{~cm})$ above the anal verge to the rectosigmoid junction; the bladder was contoured on CT in its entirety. The penile bulb was defined using CT-MRI fusion, as a pear-shaped structure comprising the proximal part of the corpus spongiosum; the femurs were defined on $\mathrm{CT}$.

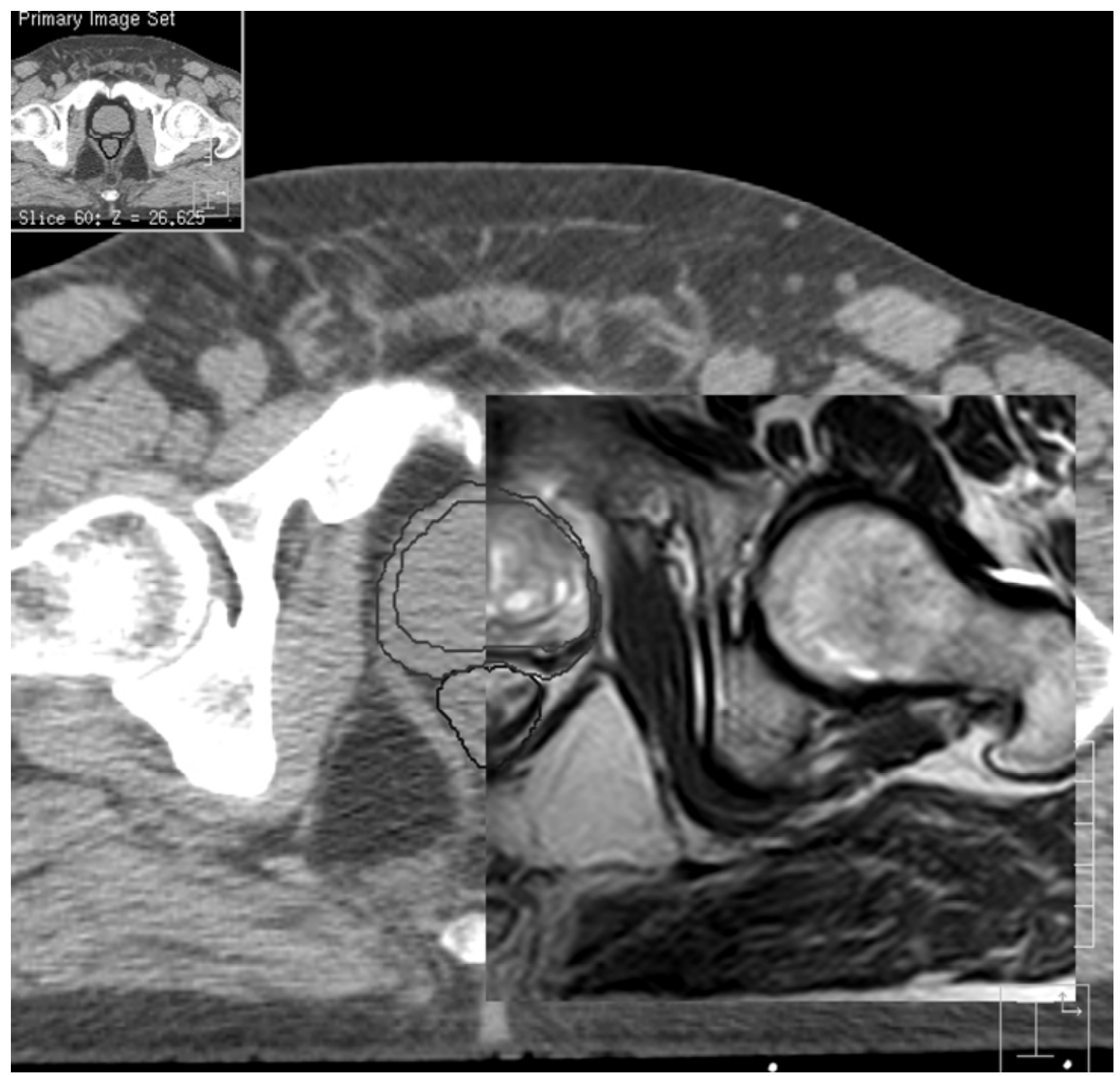

Figure 1. Axial CT- 3T MRI scan fusion. The red line is the MRI-delineated prostate contour, the purple line is the CT-delineated prostate contour and the blue line is the contour of the rectum delineated on CT-MRI fusion. 
For each patient, CT images and contours were transferred from Pinnacle to Precise Plan treatment planning system (Elekta Oncology Systems, Crawley, UK) and 3-dimension conformal radiotherapy treatment planning, with a six field arrangement, was obtained. For the purpose of this analysis, two treatment plans were generated for each patient: an MRI-plan based on the MRI-delineated CTV and a CT-plan based on the CT-delineated CTV. For an adequate PTV coverage, it was accepted that the $95 \%$ of PTV volume was covered by $95 \%$ of the prescribed dose and that the maximum dose did not exceed $107 \%$ of the prescribed dose.

The radiotherapy treatment was developed on the MRI-delineated CTVs. Daily fractions of 2 Gy ( 5 days a week) were delivered with conformal shaped treatment fields (15 MV) using the multi-leaf collimator (MLC; 1 $\mathrm{cm}$ leaf width) of an Elekta Precise linear accelerator (Elekta Precise Treatment System Plus ${ }^{\mathrm{TM}}$ ). Two orthogonal portal images were used in order to check setup alignment; digitally reconstructed radiographs (DRRs), obtained from the CT localization scans, were used as reference images; a matching software was applied to quantify set-up errors between DRRs and portal images.

For biochemical failure definition we referred to the Phoenix definition, revised by ASTRO and RTOG in Phoenix, as a rise in PSA by $2 \mathrm{ng} / \mathrm{ml}$ or more above the nadir PSA (defined as the lowest PSA achieved) [14].

Acute rectal toxicity (within 90 days from the start of radiotherapy) and late rectal toxicity were scored by the radiation oncologist, according to the RTOG / EORTC toxicity scale. Erectile function was assessed before radiotherapy and 1 year after the end of radiotherapy, by the 5-item version of the IIEF (International Index of Erectile Function) self-administrated questionnaire [15]. For rectal dose volume histogram (DVH) analysis we compared the values V70, V50 and V40 (defined as the percentage of rectum receiving at least 70, 50 and 40 Gy) obtained from the two treatment plans, the one developed on MRI prostate contour and the other on CT prostate contour.

For penile bulb we compared the mean dose to the $100 \%$ of the penile bulb, the D50, D70 and D90 (defined as the dose delivered to the $50 \%, 70 \%$ and $90 \%$ of penile bulb volume).

\section{Statistical analysis}

Statistical analysis was carried out using a commercial statistical software package (SPSS 9.0; SPSS Inc, Chicago, IL). The data were tested for normality with the Kolmogorov-Smirnov test, and different datasets were compared with paired Student's t tests (two tailed). For survival analysis Kaplan-Meier method was used.

\section{Results}

All 18 patients received 3D-conformal radiotherapy based on MRI-contoured CTVs. The median and the mean follow-up were 23.71 and 24.22 months respectively (range 15.76-37.16 months).

A G2 acute rectal toxicity was recorded in 5/18 patients and only 1 patient experienced a G2 late toxicity. At the time of analysis the 9 patients sexually active before radiotherapy were still sexually active (median IIEF score for the 9 patients: 20; range 17 - 25), but all referring a reduced ejaculation volume.

As concerns target volume analysis we considered the prostate and seminal vesicles $\left(\mathrm{CTV}_{1}\right)$, being $\mathrm{CTV}_{2}$ included in $\mathrm{CTV}_{1}$. In all patients, except one, the contoured volume of the prostate and seminal vesicles was larger on CT than on MRI (Table 2); the difference between the mean MRI and CT volumes $(58.1 \pm 27.5 \mathrm{cc}$ for MRI $\mathrm{CTV}_{1}$ vs $73.7 \pm 33.8$ cc for CT-CTV 1 ) was statistically significant $(\mathrm{p}=0.0001$, paired Student's $t$-test).

The mean volume of the rectum was $46.94 \pm 9.66 \mathrm{cc}$ (range: $31.21-63.56 \mathrm{cc}$ ); and the mean volume of the bulb of the penis was $6.93 \pm 1.79 \mathrm{cc}$ (range: $4.4 \mathrm{cc}-9.7$ cc).

Table 3 shows the rectum dose-volume-histograms (DVHs) parameters, for the total dose of $76 \mathrm{~Gy}$, from treatment plans based on CT-PTVs and on MRI-PTVs: the volume of rectum receiving a dose $\geq 70 \mathrm{~Gy}, 50 \mathrm{~Gy}$, 40 Gy is significantly reduced in MRI-plans compared to CT-plans ( $\mathrm{p}<0.05$, paired Student's $t$-test).

In penile bulb DVHs analysis, for the total dose of 76 Gy, the difference in terms of mean dose to the $100 \%$ of the volume between MRI-plans and CT-plans (39.14 Gy vs $43.86 \mathrm{~Gy})$ was not statistically significant $(\mathrm{p}=0.1$ paired Student's $t$-test), while there was a statistically significant difference in D50, D70 and D90 values ( $p<$ 0.05, paired Student's t-test), as reported in Table 4.

\section{Discussion}

Our analysis confirmed that MRI-defined CTV (prostate and seminal vesicles) is significantly smaller than the CT-defined one $(p=0.0001)$; this result is based on the better definition on MRI of the prostate, the seminal vesicles and the peri-prostatic tissues anatomy, with a reduction of the 3 major diameters (cranio-caudal; antero-posterior and latero-lateral diameter).

Several studies have demonstrated the gain in prostate volume definition using MRI, with better normal tissue sparing due to the more exact contouring of the target and to the minor interobserver variability [16-18]; these data are mainly based on $1.5 \mathrm{~T}$ MRI without endorectal 
coil. In the study of Debois et al. the average prostate volume, in 10 patients irradiated for localized prostate carcinoma, was $51 \pm 25 \mathrm{cc}$ on $\mathrm{CT}$ while on $1.5 \mathrm{~T}$ MR it was $35 \pm 17 \mathrm{cc}(\mathrm{p}=0.004$, paired Student's $t$-test $)[16]$. Rasch et al. evaluated the difference between prostate delineation on CT and MR (interscan variation) in 18 patients treated for localized prostate cancer. Three radiation oncologists delineated the prostate on CT and MRI: CT volumes were significantly larger than MRI volumes in 52 of 54 delineations [17]. The significant decrease of the interobserver delineation variability with the use of MRI has been illustrated by Villeirs et al. [18]: the retrospective analysis of prostate and seminal vesicles volume, in 13 patients, delineated by three radiation oncologists on $\mathrm{CT}$ and on $\mathrm{CT}+1 \mathrm{~T}$ MRI showed that there was a $63.06 \%$ reduction of the standard deviation around the mean CTV volume, when 1T MRI was used in addition to $\mathrm{CT}$.

Table 2. Volume comparison between MRI-defined and CT-defined $\mathrm{CTV}_{1}(\mathrm{n}=18)$.

\begin{tabular}{ccc}
\hline Patient & MRI-CTV 1 volume $(\mathrm{cc})$ & CT-CTV $_{1}$ volume $(\mathrm{cc})$ \\
\hline 1 & 93 & 129.2 \\
2 & 62.2 & 64.2 \\
3 & 44.9 & 52.6 \\
4 & 64.5 & 85.9 \\
5 & 60.6 & 73.8 \\
6 & 28.1 & 26.7 \\
7 & 108.2 & 130.8 \\
8 & 53.8 & 69.4 \\
9 & 128.9 & 150.7 \\
10 & 42 & 42.5 \\
11 & 66.4 & 99.2 \\
12 & 50 & 64.3 \\
13 & 34.8 & 39.6 \\
14 & 37.7 & 56.1 \\
15 & 49.7 & 57.4 \\
16 & 32.7 & 58.9 \\
17 & 59.7 & 75.2 \\
18 & 27.8 & 49.5 \\
& mean value (cc) \pm sd & mean value (cc) \pm sd \\
& $58.1 \pm 27.5$ & $73.7 \pm 33.8$ \\
\hline
\end{tabular}

Table 3. Rectal dose volume histogram comparison $(n=18)$. P-value refers to paired $t$-test.

\begin{tabular}{cccc}
\hline DVH parameter & mean CT volume (cc) \pm SD & mean MRI volume (cc) \pm SD & p-value \\
\hline V 70 & $16.75 \pm 5.56$ & $9.78 \pm 6.82$ & 0.0010 \\
V 50 & $36.63 \pm 7.87$ & $29.69 \pm 8.40$ & 0.0001 \\
V 40 & $43.69 \pm 8.27$ & $37.13 \pm 9.82$ & 0.0010 \\
\hline
\end{tabular}

Table 4. Penile bulb dose volume histogram comparison $(n=18)$. P-value refers to paired t-test.

\begin{tabular}{cccc}
\hline DVH parameter & CT & MRI & p-value \\
\hline Mean Dose (Gy) \pm SD & $43.86 \pm 13.16$ & $39.14 \pm 12.84$ & 0.106 \\
D90 (Gy) \pm SD & $15.38 \pm 7.8$ & $10.22 \pm 6.3$ & 0.024 \\
D70 (Gy) \pm SD & $27.33 \pm 6.11$ & $19.83 \pm 5.9$ & 0.015 \\
D50 (Gy) \pm SD & $46 \pm 16.9$ & $39 \pm 16.1$ & 0.006 \\
\hline
\end{tabular}


The use of endorectal coil in 1.5T MR prostate imaging resulted in a higher spatial resolution with a significant improvement of anatomic details [19-21]; on the other hand endorectal coils produce changes in shape and volume of the prostate, induced by the pressure on the parenchyma of the gland and may deform the peripheral zone that is typically involved in prostate cancer; the same pressure should be reproduced during the radiotherapy treatment sessions, where endorectal coil should be replaced by an endorectal balloon [22-24]. Finally endorectal coil can result in hyperintense signal intensity near the rectum, the peripheral zone and the neurovascular bundle, making the image interpretation difficult.

Some author made an image quality comparison between 3T MRI and endorectal 1.5T MRI. Sosna et al. prospectively compared 20 patients who underwent $3 \mathrm{~T}$ MRI with 20 patients who had a $1.5 \mathrm{~T}$ MRI with endorectal coil, in terms of image quality, reporting that image quality at $3 \mathrm{~T}$ without endorectal coil can be comparable with the one obtained at $1.5 \mathrm{~T}$ with an endorectal coil [25].

Park et al. compared the magnetic resonance imaging quality and local staging accuracy for prostate cancer, using phased-array 3T and endorectal 1.5T MRI. Two groups, each consisting of 54 patients, were retrospectively evaluated: one group underwent 3T MRI using a phased-array coil, the other had 1.5T MRI with endorectal coil. The incidence of MR artefacts was higher in $1.5 \mathrm{~T}$ than in 3T MRI $(\mathrm{p}=0.00)$, and 3T MRI did not show any artefact in $57 \%$ of patients, while $1.5 \mathrm{~T}$ MRI had artefacts in all patients; $1.5 \mathrm{~T}$ MRI artefacts were the hyperintense signal intensity around the rectum, image distortion from entrapped air and decreasing signal-tonoise ratio (SNR) of remote area from the coil. On the other hand, artefacts at 3T MRI, which are common to 1.5 MRI, are related to bowel peristalsis and patient motion [13].

For these reasons 3T MR image quality may be considered superior to $1.5 \mathrm{~T}$ MRI and when available may be valuable to better define prostate and seminal vesicles and normal surrounding structures without the evident disadvantages and discomfort due to the use of endorectal coil.

All these efforts obtained apparently two important results: the first one was to better define prostate, seminal vesicles and the rectum and the second one to spare normal tissue irradiation with the improvement of tolerance of the irradiation and evident reduction of rectal toxicity in particular. In this limited number of patients rectal toxicity was almost absent or limited to G2 transitory complaints disappearing within a few weeks from the end of the treatment. Sexual activity may be difficult to assess before and after treatment but the nine patients with an active sexual life before treatment declared to retain potency after the end of the irradiation. Psychological reasons might influence the sexual life of these patients besides tumour or treatment damage to the pudendal structures, but rectal toxicity $\geq \mathrm{G} 2$ is always reported as a major problem in all the patients receiving radical treatment for prostate cancer. Different methods may contribute to reduce rectal toxicity and an important one is, at least in our experience, the combination of bowel preparation with the better definition of the organ contours obtained using MR images.

The use of bowel preparation, with a diet and a daily mild laxative, ensures a reproducible rectal volume during CT and MRI acquisition and treatment session. This methodology allows the daily reproducibility of the radiation treatment, avoiding the organ motion due to the rectum repletion hence diminishing prostate dislocation $[26,27]$. In empty rectum condition the upper tract of the anterior rectal wall is far from the posterior surface of the prostate and seminal vesicles, with evident advantages in the development of the treatment plan. The use of MRI for target volume definition combined with the rectal preparation, for the daily reproducibility of rectal volume and position, allows to reduce the PTV margins, in particular towards the posterior and caudal margins, with less treatment related toxicity [28].

The analysis of rectal dose volume histograms showed a statistically significant reduction of about $40 \%$ ( $p=$ 0.001 ) of rectum receiving a dose of 70 Gy in MRI-defined treatment plans compared to CT-defined plans. The same results were demonstrated for the rectal volume receiving the dose of $40 \mathrm{~Gy}$ and $50 \mathrm{~Gy}$, where plans based on MRI-CTV showed a volume decrease of about $18 \%$ and $15 \%$ compared with plans based on CT-CTV. Our data are consistent with the results of analogous studies [16,26]; Debois et al. [16] found a statistically significant decrease of about $23.8 \%$ of the rectum receiving $80 \%$ of the prescribed dose for the treatment plans based on MRI delineation compared with the treatment plans based on CT delineation in $8 / 10$ patients investigated. Krempien et al. showed that the mean dose received by the rectum could be reduced from $74.9 \%$ to $64.2 \%$ of the prescribed dose using MRI delineation compared with CT delineation of the prostate [29].

The penile bulb is best visualized on T2-weighted MR appearing as an oval-shaped, hyper-intense midline structure under the prostatic apex. An underestimation of the prostatic apex location could lead to a geographical tumour miss, while an overestimation may cause erectile dysfunction due to irradiation of the penile bulb; MRI help to discriminate between the prostate apex and the proximal penile bulb. Erectile dysfunction is a long-term sequela after definitive radiotherapy for prostate cancer 
[30]; the irradiation of the proximal penile tissue damage vascular and nervous structures supplying the cavernosus muscle [31]. Merrik et al. [32] demonstrated that if the dose delivered with brachytherapy to $50 \%$ of the bulb was $<50 \mathrm{~Gy}$, potency was likely to be conserved. In the analysis of Fisch et al. a dose to $50 \%$ of the bulb $<48.5$ Gy was associated with no risk of erectile dysfunction [31]. In the recent issue by Roach et al. [33] it is suggested to keep the mean dose to the $95 \%$ of the bulb under $50 \mathrm{~Gy}$.

In our experience the bulb of the penis received significant less dose with the treatment plans based on MRI-delineated CTV compared with those based on the CT-delineated CTV. We found that the mean value of D 50 was $46 \pm 16.9$ Gy in CT-plans radiotherapy versus 39 \pm 16.1 Gy in MRI-plans $(p=0.006)$.

In conclusion in this preliminary experience we proved the feasibility of CT-3T MRI image registration under radiotherapy planning conditions. The results of our analysis showed that 3T MRI improves the definition of the target volume sparing normal tissue irradiation.

\section{References}

[1] A. Jemal, R. Siegel, E. Ward, Y. Hao, J. Xu and M. J. Thun, "Cancer Statistics, 2009," A Cancer Journal for Clinicians, Vol. 59, No. 4, 2009, pp. 225-249. doi:10.3322/caac.20006

[2] J. Ferlay, P. Autier, M. Boniol, M. Heanue, M. Colombet and P. Boyle, "Estimates of Cancer Incidence and Mortality in Europe in 2006," Annals of Oncology, Vol. 18, No. 4, 2007, pp. 581-592. doi:10.1093/annonc/mdl498

[3] R. E. Peschel and J. W. Colberg, "Surgery, Brachytherapy, and External-Beam Radiotherapy for Early Prostate Cancer," The Lancet Oncology, Vol. 4, 2003, pp. 233-241. doi:10.1016/S1470-2045(03)01035-0

[4] A. V. D'Amico, R. Whittington and I. Kaplan, et al., "Equivalent Biochemical Failure-Free Survival after External Beam Radiation Therapy or Radical Prostatectomy in Patients with a Pretreatment Prostate Specific Antigen of > 4-20 ng/ml," International Journal of Radiation Oncology Biology Physics, Vol. 37, 1997, pp. 1053-1058.

doi:10.1016/S0360-3016(96)00633-5

[5] A. V. D'Amico, R. Whittington and S. B. Malkowicz, et al., "Biochemical outcome after radical prostatectomy, external beam radiation therapy, or interstitial radiation therapy for clinically localized prostate cancer," The Journal of the American Medical Association, Vol. 280, 1998, pp. 969-974.

doi:10.1001/jama.280.11.969

[6] L. Potters, E. A. Klein and M. W. Kattan, et al., "Monotherapy for Stage T1-T2 Prostate Cancer: Radical Prostatectomy, External Beam Radiotherapy, or Permanent
Seed Implantation," Radiotherapy and Oncology, Vol. 71, 2004, pp. 29-33.

doi:10.1016/j.radonc.2003.12.011

[7] A. A. Aizer, J. B. Yu, J. W. Colberg, A. M. McKeon, R. H. Decker and R. E. Peschel, "Radical Prostatectomy vs. Intensity-Modulated Radiation Therapy in the Management of Localized Prostate Adenocarcinoma," Radiotherapy and Oncology, Vol. 93, No. 2, 2009, pp. 185-191. doi:10.1016/j.radonc.2009.09.001

[8] S. Welz, M. Nyazi, C. Belka and U. Ganswindt, "Surgery vs. Radiotherapy in Localized Prostate Cancer. Which is best?” Radiation Oncology, Vol. 7, No. 3, 2008, p. 23. doi:10.1186/1748-717X-3-23

[9] M. J. Zelefsky, Z. Fuks and M. Hunt, et al., "High-dose intensity modulated radiation therapy for prostate cancer: early toxicity and biochemical outcome in 772 patients," International Journal of Radiation Oncology Biology Physics, Vol. 53, No. 5, 2002, pp. 1111-1116. doi:10.1016/S0360-3016(02)02857-2

[10] J. M. Michalski, K. Bae and M. Roach, et al., "Long-Term Toxicity Following 3D Conformal Radiation Therapy for Prostate Cancer from the RTOG 9406 Phase I /II Dose Escalation Study," International Journal of Radiation Oncology Biology Physics, Vol. 76, No. 1, 2010, pp. 14-22. doi:10.1016/j.ijrobp.2009.01.062

[11] L. Perna, C. Fiorino and C. Cozzarini, et al., "Sparing the Penile Bulb in the Radical Irradiation of Clinically Localised Prostate Carcinoma: A Comparison between MRI and CT Prostatic Apex Definition in 3DCRT, Linac-IMRT and Helical Tomotherapy," Radiotherapy and Oncology, Vol. 93, No. 1, 2009, pp. 57-63. doi:10.1016/j.radonc.2009.04.004

[12] A. Villers, L. Lemaitre, J. Haffner and P. Puech, "Current Status of MRI for the Diagnosis, Staging and Prognosis of Prostate Cancer: Implications for Focal Therapy and Active Surveillance," Current Opinion in Urology, Vol. 19, No. 3, 2009, pp. 274-282. doi:10.1097/MOU.0b013e328329a2ed

[13] B. K Park, B. Kim and C. K. Kim, et al, "Comparison of Phased-Array 3.0-T and Endorectal 1.5-T Magnetic Resonance Imaging in the Evaluation of Local Staging Accuracy for Prostate Cancer," Journal of Computer Assisted Tomography, Vol. 31, No. 4, 2007, 534-538. doi:10.1097/01.rct.0000250108.85799.e1

[14] M. Roach $3^{\text {rd }}$, G. Hanks and H. Thames, et al, "Defining Biochemical Failure Following Radiotherapy with or without Hormonal Therapy in Men with Clinically Localized Prostate Cancer: Recommendations of the RTOG-ASTRO Phoenix Consensus Conference," International Journal of Radiation Oncology Biology Physics, Vol. 65, 2006, pp. 965-974. doi:10.1016/j.ijrobp.2006.04.029

[15] R. C. Rosen, J. C. Cappelleri and M. D. Smith, et al., "Development and Evaluation of an Abridged, 5-Item Version of the International Index of Erectile Function (IIEF-5) as a Diagnostic Tool for Erectile Dysfunction," International Journal of Impotence Research, Vol. 11, 1999, pp. 319-326. doi:10.1038/sj.ijir.3900472

[16] M. Debois, R. Oyen and F. Maes, et al., "The Contribu- 
tion of Magnetic Resonance Imaging to the Three-Dimensional Treatment Planning of Localized Prostate Cancer," International Journal of Radiation Oncology Biology Physics, Vol. 45, No. 4, 1999, pp. 857-865. doi:10.1016/S0360-3016(99)00288-6

[17] C. Rasch, I. Barillot, P. Remeijer, A. Touw, M. van Herk, J. V. Lebesque, "Definition of the prostate in CT and MRI: a multi-observer study," International Journal of Radiation Oncology Biology Physics, Vol. 43, No. 1, 1999, pp. 57-66. doi:10.1016/S0360-3016(98)00351-4

[18] G. M. Villeirs, K. van Vaerenbergh, L. Vakaet, et al., "Interobserver delineation variation using CT + MRI in intensity-modulated radiotherapy for prostate cancer," Strahlentherapie und Onkologie, Vol. 181, No. 7, 2005, pp. 424-430. doi:10.1007/s00066-005-1383-x

[19] M. L. Schiebler, M. D. Schnall and H. M. Pollack, et al., "Current Role of MR Imaging in the Staging of Adenocarcinoma of the Prostate," Radiology, Vol. 189, No. 2, 1993, pp. 339-352. PMid:8210358

[20] H. Hricak, S. White and D. Vigneron, et al., "Carcinoma of the Prostate Gland: MR Imaging with Pelvic PhasedArray Coils Versus Integrated Endorectal-Pelvic PhasedArray Coils," Radiology, Vol. 193, No. 3, 1994, pp. 703709. PMid:7972810

[21] J. J. Futterer, M. R. Engelbrecht and G. J. Jager, et al., "Prostate Cancer: Comparison of Local Staging Accuracy of Pelvic Phased-Array Coil Alone Versus Integrated Endorectal-Pelvic Phased-Array Coils: Local Staging Accuracy of Prostate Cancer Using Endorectal Coil MR Imaging," European Radiology, Vol. 17, 2007, pp. 10551065.PMid:1- 7024497

[22] J. E. Husband, A. R. Padhani and A. D. MacVicar, et al., "Magnetic Resonance Imaging of Prostate Cancer: Comparison of Image Quality Using Endorectal and Pelvic Phased Array Coils," Clinical Radiology, Vol. 53, 1998, pp. 673-681. doi:10.1016/S0009-9260(98)80294-8

[23] Y. Kim, I. C. Hsu and J. Pouliot, et al., "Expandable and Rigid Endorectal Coils for Prostate MRI: Impact on Prostate Distortion and Rigid Image Registration," Medical Physics, Vol. 32, 2005, pp. 3569-3578. doi:10.1118/1.2122467

[24] S. W. Heijmink, T. W. Scheenen, E. N. V. Lin, A. G. Visser, L. A. Kiemeney, J. A. Witjes and J. O. Barentsz, "Changes in Prostate Shape and Volume and Their Implications for Radiotherapy after Introduction of Endorectal Ballon as Determined by MRI at 3T," International Journal of Radiation Oncology Biology Physics, Vol. 73, No. 5, 2009, pp. 1446-1453. doi:10.1016/j.ijrobp.2008.06.1491

[25] J. Sosna, I. Pedrosa, W. C. Dewolf, H. Mahallati, R. E. Lenkinski and N. M. Rofsky, "MR Imaging of the Pros- tate at 3 Tesla: Comparison of an External Phased-Array Coil to Imaging with an Endorectal Coil at 1.5 Tesla," Academic Radiology, Vol. 11, No. 8, 2004, pp. 857-862. doi:10.1016/j.acra.2004.04.013

[26] C. Fiorino, N. Di Muzio and S. Broggi, et al., "Evidence of Limited Motion of the Prostate by Carefully Emptying the Rectum as Assessed by Daily MVCT Image Guidance with Helical Tomotherapy," International Journal of Radiation Oncology Biology Physics, Vol. 71, No. 2, 2008, pp. 611-617. doi:10.1016/j.ijrobp.2008.01.048

[27] M. Stasi, F. Munoz and C. Fiorino, et al., "Emptying the Rectum before Treatment Delivery Limits the Variations of Rectal Dose-Volume Parameters During 3DCRT of Prostate Cancer," Radiotherapy and Oncology, Vol. 80, No. 3, 2006, pp. 363-370.

doi:10.1016/j.radonc.2006.08.007

[28] R. J. Steenbakkers, K. E. Deurloo, P. J. Nowak, J. V. Lebesque, M. V. Herk and C. R. Rasch, "Reduction of the Dose Delivered to the Rectum and Bulb of the Penis Using MRI Delineation for Radiotherapy of the Prostate," International Journal of Radiation Oncology Biology Physics, Vol. 57, No. 5, 2003, pp. 1269-79. doi:10.1016/S0360-3016(03)01446-9

[29] R. C. Krempien, K. Schubert and D. Zierhut, et al., "Open Low-Field Magnetic Resonance Imaging in Radiation Therapy Treatment Planning," International Journal of Radiation Oncology Biology Physics, Vol. 53, No. 5, 2002, pp. 1350-1360. doi:10.1016/S0360-3016(02)02886-9

[30] K. E. Wallner, G. S. Merrick, M. L. Benson, W. M. Butler, J. Maki and B. G. Tollenaar, "Penile Bulb Imaging," International Journal of Radiation Oncology Biology Physics, Vol. 53, No. 4, 2002, pp. 928-933. doi:10.1016/S0360-3016(02)02805-5

[31] B. M. Fish, B. Pickett and V. Weinberg, et al., "Dose of Radiation Received by the Bulb of the Penis Correlates with the Risk of Impotence after Three-Dimensional Conformal Radiotherapy for Prostate Cancer," Urology, Vol. 57, 2001, pp. 955-959. doi:10.1016/S0090-4295(01)00940-2

[32] G. S. Merrik, K. Wallner and W. M. Butler, et al., "A Comparison of Radiation Dose to the Bulb of the Penis in Men with and without Prostate Brachytherapy-Induced Erectile Dysfunction," International Journal of Radiation Oncology Biology Physics, Vol. 50, 2001, pp. 597-604. doi:10.1016/S0360-3016(01)01475-4

[33] M. Roach 3rd, J. Nam, G. Gagliardi, I. El Naqa, J. O. Deasy and L. B. Marks, "Radiation Dose-Volume Effects and the Penile Bulb," International Journal of Radiation Oncology Biology Physics, Vol. 76, No. 3, 2010, pp. S130-S134. doi:10.1016/j.ijrobp.2009.04.094 